\title{
WEAK REPRESENTATIONS OF RELATION ALGEBRAS AND RELATIONAL BASES
}

\author{
ROBIN HIRSCH, IAN HODKINSON, AND ROGER D. MADDUX
}

\begin{abstract}
It is known that for all finite $n \geq 5$, there are relation algebras with $n$-dimensional relational bases but no weak representations. We prove that conversely, there are finite weakly representable relation algebras with no $n$-dimensional relational bases. In symbols: neither of the classes RA And $_{n}$ aRRA contains the other.
\end{abstract}

The main result of this paper concerns the relationship between two classes each of which contains the class of representable relation algebras (RRA). Representable relation algebras are isomorphic to genuine fields of binary relations with natural set-theoretically defined operators (union, set complement, identity, converse and composition). It is known that RRA is badly behaved in a number of ways. RRA cannot be defined by finitely many axioms [14], nor by any set of equations using only finitely many variables [8], nor by any set of first-order sentences containing only finitely many non-canonical sentences [6]. It is undecidable whether a finite relation algebra is representable or not [4]. The equational theory of RRA is undecidable [16]. Consequently, researchers have defined and investigated other classes of relation algebras, not identical to RRA but with some common features.

One of these classes is the class of weakly representable relation algebras (wRRA), which have representations rather like classical representations, but the Boolean sum and negation operators are not required to be interpreted as set union and complement respectively [7, page 459]. Clearly wRRA $\supseteq$ RRA and it turns out that the inclusion is proper.

Another kind of representation which allows us to generalise RRA is called a relativised representation. The definition is like the definition of a classical representation, but all operators are relativised to the unit element in the representation, which is simply a reflexive and symmetric binary relation containing all the other relations. The class of relation algebras with relativised representations turns out to be very well behaved - all relation algebras have relativised representations, the class of relation-type algebras with relativised representations is finitely axiomatisable [10, Theorem 5.20], and all finite relation algebras have finite relativised representations [4, Theorem 19.13]. There are many variants of this definition which impose different restrictions on the type of unit that is allowed. A set of points in the

Received October 13, 2009.

2010 Mathematics Subject Classification. 03G15. 
domain of a relativised representation is called a clique if every pair of points from the set belongs to the unit of the representation. A special kind of relativised representation, called an $n$-square representation, has the following property: for any clique $C$ with fewer than $n$ points, and any pair of points from the clique, belonging to the representation of a composition of two elements from the algebra, $C$ is contained in some clique that contains a point witnessing the composition. The class of relation algebras with $n$-square representations is called $\mathrm{RA}_{n}$ (though the standard definition of this class is given in terms of bases: see below). Although $\mathrm{RA}_{\omega}=\mathrm{RRA}$ (see [11, Theorem 6] or [13, Theorem 418]), RA ${ }_{n}$ is quite well-behaved for finite $n$. Every finite relation algebra in $\mathrm{RA}_{n}$ has a finite $n$-square representation [4, Theorem 19.18] and a finite $n$-dimensional relational basis [13, Theorems 411,325], and it follows that the problem of determining whether a finite relation algebra belongs to $\mathrm{RA}_{n}$ is decidable. The classes $\mathrm{RA}_{n}: n<\omega$ form a sequence of better and better approximations to RRA in the sense that $R A=R A_{4} \supset R A_{5} \supset R A_{6} \supset \cdots$ (these containments are strict [12] —indeed, for $n \geq 4, \mathrm{RA}_{n+1}$ is not finitely axiomatisable over $\mathrm{RA}_{n}[4$, Theorem 17.18]) and

$$
\bigcap_{4 \leq n<\omega} \mathrm{RA}_{n}=\mathrm{RA}_{\omega}=\mathrm{RRA} .
$$

These two kinds of generalisation of classical representability have very different definitions and each class has its strengths and weaknesses. For quite some time it was not known whether wRRA contained $\mathrm{RA}_{n}$ (any $n$ with $5 \leq n<\omega$ ) nor whether $\mathrm{RA}_{n}$ contained wRRA. Jónsson [7, problem 3] asked whether the inclusion wRRA $\supseteq$ RRA is strict, and Andréka [1] proved that it is, but the algebras she constructs turn out to belong to $R A_{5}$ and $R A_{6}$. Of course, each of Andréka's algebras must fail to belong to $\mathrm{RA}_{n}$ for some $n<\omega$ by (1), since they have no classical representations, and therefore $\mathrm{wRRA} \nsubseteq \mathrm{RA}_{n}$, for some $n<\omega$, but we do not know the least value of $n$ such that one of her weakly representable algebras is not in $\mathrm{RA}_{n}$, and this least value is certainly greater than six, so her construction does not tell us exactly how these classes relate to each other.

Maddux suggested that the relationship between $\mathrm{RA}_{n}$ and wRRA should be clarified, and in particular he asked about the relationship between $R A_{5}$ and wRRA. This problem was stated as [4, problem 18.26]. In [4, Corollary 18.25] it was shown that $\mathrm{RA}_{5} \nsubseteq \subseteq$ wRRA (and indeed that RA $\mathrm{R}_{n} \nsubseteq \subseteq$ wRRA for every finite $n \geq 3$ ). In this paper, we prove that there are weakly representable relation algebras not belonging to $\mathrm{RA}_{5}$ (hence not belonging to $\mathrm{RA}_{n}$ for $5 \leq n<\omega$ ). So neither wRRA nor $\mathrm{RA}_{5}$ contains the other.

We will say a bit more about these classes after we have given the formal definitions.

Definition 1 (Tarski). A relation algebra $\mathscr{A}=\left(A, 0,1,+, \cdot,-, 1^{\prime},-, ;\right)$ is a Boolean algebra $(A, 0,1,+, \cdot,-)$ together with a constant $1^{\prime} \in A$, a unary function ${ }^{-}$, and a binary function;, such that

- $\left(A, ;, 1^{\prime}\right)$ is a monoid,

- $(a ; b) \cdot c=0 \Longleftrightarrow(\breve{a} ; c) \cdot b=0 \Longleftrightarrow a \cdot(c ; \breve{b})=0$, for all $a, b, c \in A$ (the so-called 'Peircean law').

The class of all relation algebras is denoted RA. 
Definition 2 (Jónsson). Let $\mathscr{A}$ be a relation algebra. A weak representation $h$ over the base set $X$ is a $1-1$ map $h: \mathscr{A} \rightarrow \wp(X \times X)$ such that for all elements $a, b$ of $\mathscr{A}$, the following hold:

$$
\begin{aligned}
h(0) & =\emptyset, \\
h(a \cdot b) & =h(a) \cap h(b), \\
h\left(1^{\prime}\right) & =\{(x, x): x \in X\}, \\
h(\breve{a}) & =\{(x, y):(y, x) \in h(a)\}, \\
h(a ; b) & =\{(x, y): \exists z((x, z) \in h(a) \wedge(z, y) \in h(b))\} .
\end{aligned}
$$

The class wRRA is defined to be the class of relation algebras with weak representations.

Until recently, not much was known about wRRA. The class is clearly closed under subalgebras and direct products, and by [15] we now know that this class is also closed under homomorphic images and therefore forms an equational variety. But it is not finitely axiomatisable [2, 3, 5], and there is no algorithm for determining whether a finite relation algebra has a weak representation [4]. Indeed, we have:

THEOREM 3. [4, Theorem 18.23] If $K$ is any class of relation algebras such that $\mathrm{RRA} \subseteq K \subseteq$ wRRA then the problem of determining whether an arbitrary finite relation algebra belongs to $K$ is undecidable.

So in one sense, RRA and wRRA are very close: restricted to finite algebras, RRA and (the complement of) wRRA are recursively inseparable.

We now come to the classes $\mathrm{RA}_{n}$. To define them, we need to consider atoms and networks. Let $\mathscr{A}$ be a relation algebra. We can define a Boolean ordering by $x \leq y \Longleftrightarrow x+y=y$. An atom of $\mathscr{A}$ is a minimal non-zero element in this ordering. We write $A t(\mathscr{A})$ for the set of atoms of $\mathscr{A} . \mathscr{A}$ is atomic if every non-zero element is above some atom. Because $(x \mapsto \breve{x})$ is an automorphism of the Boolean reduct of $\mathscr{A}$, the converse of an atom is an atom.

DEFINITION 4 (Lyndon). Let $\mathscr{A}$ be an atomic relation algebra. The atom structure $\mathfrak{A} \mathfrak{t}(\mathscr{A})=\left(A t(\mathscr{A}), I d,{ }^{`}, C\right)$ of $\mathscr{A}$ is a four-tuple where $I d$ is the set of atoms below the identity of $\mathscr{A},-$ is the restriction of the converse operator of $\mathscr{A}$ to atoms, and $C$ is the set of triples of atoms $(a, b, c)$ such that $a ; b \geq c$.

The Peircean transforms of a triple of atoms $(a, b, c)$ are

$$
(a, b, c),(b, \breve{c}, \breve{a}),(\breve{c}, a, \breve{b}),(\breve{b}, \breve{a}, \breve{c}),(\breve{a}, c, b),(c, \breve{b}, a) .
$$

It follows from the relation algebra axioms that if $(a, b, c) \in C$, then all Peircean transforms of $(a, b, c)$ also belong to $C$.

Conversely, given a four-tuple $\mathcal{S}=\left(A, I d,{ }^{\complement}, C\right)$, where $I d \subseteq A,{ }^{-}: A \rightarrow A$ and $C \subseteq A \times A \times A$, we define the complex algebra $\mathfrak{C m}(\mathcal{S})$ to be the algebra $(\wp(A), \emptyset, A, \cup, \cap, \backslash, I d, \breve{\sim}, ;)$ where $\breve{S}=\{\breve{s}: s \in S\}$ for any $S \subseteq A$, and $S ; T=$ $\{u \in A: \exists s \in S, t \in T,(s, t, u) \in C\}$ for any $S, T \subseteq A$.

For a finite relation algebra $\mathscr{A}$ we have $\mathfrak{C m}(\mathfrak{A t}(\mathscr{A})) \cong \mathscr{A}($ see $[9, \S 4])$, so we can define $\mathscr{A}$, up to isomorphism, by defining its atom structure.

The following definition differs from the definition of a relation algebra network [4, Definition 7.1], where we only require that $N_{2}(x, y) ; N_{2}(y, z) \cdot N_{2}(x, z) \neq 0$ and 
the definition of network inclusion is also weaker, but the stricter definition here can be used to find sufficient conditions for weak representability.

Definition 5. Let $\mathscr{A}$ be a relation algebra. A network over $\mathscr{A}$ is a pair $\left(N_{1}, N_{2}\right)$, where $N_{1}$ is a set (of 'nodes') and $N_{2}: N_{1} \times N_{1} \rightarrow \mathscr{A}$ is a function such that

$$
\begin{aligned}
N_{2}(x, x) & \leq 1^{\prime}, \\
N_{2}(y, x) & =N_{2}(x, y)^{-}, \\
N_{2}(x, y) ; N_{2}(y, z) & \geq N_{2}(x, z)
\end{aligned}
$$

for $x, y, z \in N_{1}$. The network is said to be strict if $N_{2}(x, y) \leq 1^{\prime} \Rightarrow x=y$. Observe that if $N_{2}(x, y) \neq 0$ for some $x, y \in N_{1}$ then by the third network condition $N_{2}(w, z) \neq 0$ for all $w, z \in N_{1}$.

Let $\left(M_{1}, M_{2}\right),\left(N_{1}, N_{2}\right)$ be networks. We write $\left(M_{1}, M_{2}\right) \leq\left(N_{1}, N_{2}\right)$ if $M_{1} \subseteq N_{1}$ and $N_{2}\left\lceil_{M_{1} \times M_{1}}=M_{2}\right.$. If $\left(N_{1}^{0}, N_{2}^{0}\right) \leq\left(N_{1}^{1}, N_{2}^{1}\right) \leq \cdots \leq\left(N_{1}^{n}, N_{2}^{n}\right) \leq \cdots$ is a countable sequence of nested networks then we define the limit $\bigcup_{n<\omega}\left(N_{1}^{n}, N_{2}^{n}\right)=$ $\left(N_{1}, N_{2}\right)$ by $N_{1}=\bigcup_{n<\omega} N_{1}^{n}$ and for any $x, y \in N_{1}$ we let $N_{2}(x, y)=N_{2}^{n}(x, y)$ for any $n<\omega$ such that $x, y \in N_{1}^{n}$ (since the networks are nested this is well-defined).

Now suppose that $\mathscr{A}$ is atomic. Let $N=\left(N_{1}, N_{2}\right)$ be a network over $\mathscr{A} . N$ is said to be atomic if $N_{2}(x, y) \in A t(\mathscr{A})$ for every $x, y \in N_{1}$. (Sometimes these networks have been called basic matrices.) An atomic refinement of $N$ is a pair $\left(N_{1}, N_{2}^{a}\right)$ such that $N_{2}^{a}(x, y) \in A t(\mathscr{A})$ and $N_{2}^{a}(y, x)^{-}=N_{2}^{a}(x, y) \leq N_{2}(x, y)$ for every $x, y \in N_{1}$; it may or may not be a network.

In this paper we drop the subscripts and use $N$ to refer to the network $\left(N_{1}, N_{2}\right)$, the set of nodes $N_{1}$ and the labelling $N_{2}$, distinguishing these uses by context.

Definition 6 (Maddux, $[13, \S 6.21, \S 6.24]$ ). Let $\mathscr{A}$ be an atomic relation algebra and $4 \leq n<\omega .{ }^{1}$ An $n$-dimensional relational basis for $\mathscr{A}$ is a set $B$ of atomic networks over $\mathscr{A}$ with nodes $\{0,1, \ldots, n-1\}$ and such that

- for all $a \in A t(\mathscr{A})$ there is $N \in B$ with $N(0,1)=a$, and

- for all $N \in B, i, j, k<n$ and $a, b \in A t(\mathscr{A})$, if $k \notin\{i, j\}$ and $a ; b \geq N(i, j)$ then there is $M \in B$ with $M(x, y)=N(x, y)$ for every $x, y \in\{0, \ldots, n-1\} \backslash\{k\}$ (we write $M \equiv_{k} N$ for this), $M(i, k)=a$, and $M(k, j)=b$.

The class $\mathrm{RA}_{n}$ is defined to be the closure under subalgebras of the class of atomic relation algebras with $n$-dimensional relational bases.

This definition turns out to be equivalent to the sketched definition we gave in the introduction (see [4, Theorem 13.46] for a more precise formulation and proof of this). $\mathrm{RA}_{n}$ has at least two positive features: it is a canonical variety (by [11, Theorems 8,9] or [13, Theorems 414, 420]), and there is an algorithm that determines whether a finite relation algebra belongs to this class or not (by [13, Theorems 411, 325]). This latter property is also a consequence of the following easy lemma.

Lemma 7. Let $4 \leq n<\omega$ and let $\mathscr{A} \subseteq \mathscr{B}$ be atomic relation algebras. If $\mathscr{A}$ is finite and $\mathscr{B}$ has an $n$-dimensional relational basis then so does $\mathscr{A}$.

\footnotetext{
${ }^{1} \mathrm{RA}_{2}$ and $\mathrm{RA}_{3}$ can be defined as well [13, §6.24], but we are not concerned with these cases here.
} 
Hence, a finite relation algebra $\mathscr{A}$ belongs to $\mathrm{RA}_{n}$ if and only if $\mathscr{A}$ has an $n$ dimensional relational basis (no need to worry about the case where $\mathscr{A}$ has no basis but an extension of $\mathscr{A}$ does). From this, a non-deterministic algorithm to test whether $\mathscr{A} \in \mathrm{RA}_{n}$ picks an arbitrary set $B$ of maps $f:\{0, \ldots, n-1\}^{2} \rightarrow \operatorname{At}(\mathscr{A})$ (there are only finitely many such maps, in fact $O\left(|A t(\mathscr{A})|^{n^{2}}\right)$ ), checks to see if each map in $B$ is a network, and then checks whether $B$ forms a relational basis.

The fact that we can tell by an algorithm whether a finite relation algebra is in $\mathrm{RA}_{n}$ distinguishes these classes from wRRA. Since RA $\mathrm{A}_{n} \supseteq \mathrm{RRA}$, it follows from Theorem 3 above that $\mathrm{RA}_{n}$ cannot be contained in wRRA, for any $n$ with $5 \leq n<\omega$. $^{2}$ Indeed, the undecidability proof for Theorem 3 yields an example of a finite relation algebra with a five-dimensional relational basis but no weak representation. If $\tau$ is a non-tiling instance of the deterministic tiling problem with the property that for each $t \in \tau$ there are four tiles in $\tau$ that match $t$ on the left, right, top and bottom respectively, then it can be checked that the relation algebra $R A(\tau)$ of [4, section 18.3] is not weakly representable but does have a five-dimensional relational basis. So we have:

Proposition 8. [4, Corollary 18.25] For all $n \geq 5$, there are finite relation algebras in $\mathrm{RA}_{n} \backslash$ wRRA.

The remaining problem, then, is to show that the other inclusion failswRRA $\nsubseteq \mathrm{RA}_{n}$, for $n \geq 5$, and this is the problem we tackle in the remainder of this paper. We will need to show that the algebra we construct is weakly representable. To that end, we now define a game to establish weak representability.

Definition 9. Let $\mathscr{A}$ be a relation algebra. The two player game $\mathscr{G}(\mathscr{A})$ has $\omega$ rounds. A play of the game consists of a countable sequence of strict networks

$$
N_{0} \leq N_{1} \leq N_{2} \leq \cdots
$$

In the initial round, $\forall$ picks a non-zero $a_{0} \in \mathscr{A}$ and $\exists$ has to play a strict network $N_{0}$ containing nodes $x_{0}, y_{0}$, say, such that $N_{0}\left(x_{0}, y_{0}\right)=a_{0}$. In round $k>0$ of the play, $N_{k-1}$ has been defined in the previous round, $\forall$ picks nodes $m, n \in N_{k-1}$ and elements $Y, Z \in \mathscr{A}$ such that $Y ; Z \geq N_{k-1}(m, n)$. We denote this move as $(m, n, Y, Z) . \exists$ is required to respond with a strict network $N_{k} \geq N_{k-1}$ containing a node $p$ such that $N_{k}(m, p) \leq Y$ and $N_{k}(p, n) \leq Z$. If, in some round, she fails to provide a suitable extension network then she loses the play. Otherwise, she wins.

The main difference between this game and the classical representation games of [4, chapter 7] is that the labels of edges of networks are not refined, and indeed in each round when $\exists$ extends the previous network she is not allowed to refine the labels on edges. This is essentially because a weak representation is not required to respect + or - .

Proposition 10. Let $\mathscr{A}$ be a countable relation algebra. If $\exists$ has a winning strategy in the game $\mathscr{G}(\mathscr{A})$ then $\mathscr{A}$ is weakly representable.

To prove the proposition, suppose $\exists$ has a winning strategy in the game. For each non-zero $a \in \mathscr{A}$ let $N_{0} \leq N_{1} \leq \cdots$ be a play of the game in which $\forall$ plays $a$ initially and schedules all possible moves into the subsequent play of the game-i.e., if $i<\omega$, $m, n \in N_{i}$ and $U ; V \geq N_{i}(m, n)$ then eventually $\forall$ plays $(m, n, U, V)$ - and $\exists$ uses her

${ }^{2}$ It also holds for $n=4$, but that was known from the beginning [7]. 
winning strategy. Since the networks are finite and $\mathscr{A}$ is countable, this scheduling can be done. Let $N^{a}$ be the limit of this play. By renaming nodes we can arrange that the nodes of $N^{a}$ and $N^{b}$ are disjoint, when $a \neq b$. Let $X$ be the disjoint union of the sets of nodes of the $N^{a} \mathrm{~s}$, as $a$ ranges over all non-zero elements of $\mathscr{A}$. Define a map $h: \mathscr{A} \rightarrow \wp(X \times X)$ by $h(b)=\left\{(x, y): \exists a \in \mathscr{A}\left(a>0 \wedge x, y \in N^{a} \wedge N^{a}(x, y) \leq b\right)\right\}$. It is easy to check that $h$ is a weak representation.

Problem 11 . Let $\mathscr{A}$ be a relation algebra and define a weak complete representation $h$ of $X$ to be a weak representation such that for any subset $S$ of $\mathscr{A}$ whose infimum $\inf (S)$ exists in $\mathscr{A}$ we have $h(\inf (S))=\bigcap\{h(s): s \in S\}$. Is it the case that every weakly representable relation algebra has a weak complete representation? If not, is the class of relation algebras with weak complete representations an elementary class? If $\mathscr{A}$ has a weak complete representation, must $\exists$ have a winning strategy in $\mathscr{G}(\mathscr{A})$ ?

We will now construct an algebra $\mathscr{A}_{0}$, a kind of 'rainbow algebra' (cf. [4, Chapter 16]), and show it is a weakly representable relation algebra but is not in $\mathrm{RA}_{5}$.

Definition 12. We define a finite algebra $\mathscr{A}_{0}$, which we will see in Lemma 13 to be a relation algebra, by defining its atom structure. The set of atoms $A t$ is

$$
\left\{1^{\prime}, \mathrm{g}, \mathrm{g}^{\prime}, \mathrm{y}, \mathrm{y}^{\prime}, \mathrm{b}, \breve{\mathrm{b}}, \mathrm{w}_{i}, \mathrm{~s}, \mathrm{v}, \alpha_{i}, \beta_{i}, \mathrm{r}_{i}, \breve{\mathrm{r}}_{i}: i<2\right\} .
$$

There are 19 atoms here. It may help to think of $g, g^{\prime}$ as green, $y, y^{\prime}$ as yellow, $b, \breve{b}$ as black, $w_{i}$ as white, $s$ as silver, $v$ as violet, and $r_{i}, \breve{r}_{i}$ as red. The only atom below the identity is $1^{\prime}$ itself. The atoms $1^{\prime}, \mathrm{v}, \mathrm{g}, \mathrm{g}^{\prime}, \mathrm{y}, \mathrm{y}^{\prime}, \mathrm{w}_{i}, \mathrm{~s}, \alpha_{i}, \beta_{i}$ are self-converse and the other atoms form converse pairs: $(\mathrm{b}, \breve{\mathrm{b}})$ and $\left(\mathrm{r}_{i}, \breve{r}_{i}\right)$ for $i<2$. Let

$$
\begin{aligned}
& \mathrm{A}=\mathrm{g}+\mathrm{g}^{\prime}+\mathrm{y}+\mathrm{y}^{\prime}+\sum_{i<2}\left(\alpha_{i}+\beta_{i}\right), \\
& \mathrm{E}=\mathrm{b}+\breve{\mathrm{b}}+\mathrm{s}+\sum_{i<2}\left(\mathrm{w}_{i}+\mathrm{r}_{i}+\breve{\mathrm{r}}_{i}\right), \\
& \Gamma=\mathrm{g}+\mathrm{g}^{\prime}+\mathrm{y}+\mathrm{y}^{\prime}, \\
& \mathrm{R}=\mathrm{r}_{0}+\mathrm{r}_{1} .
\end{aligned}
$$

Note that $\Gamma \leq \mathrm{A}$ and $1=1^{\prime}+\mathrm{E}+\mathrm{A}+\mathrm{v}$. Atoms will be denoted by lower case variables and arbitrary elements of $\mathscr{A}_{0}$ by upper case variables. For example, if we write $a \leq \mathrm{A}$ it will be implicit that $a$ is an atom.

The forbidden triples (i.e., atoms $(a, b, c)$ such that $a ; b \cdot c=0)$ are the Peircean transforms of

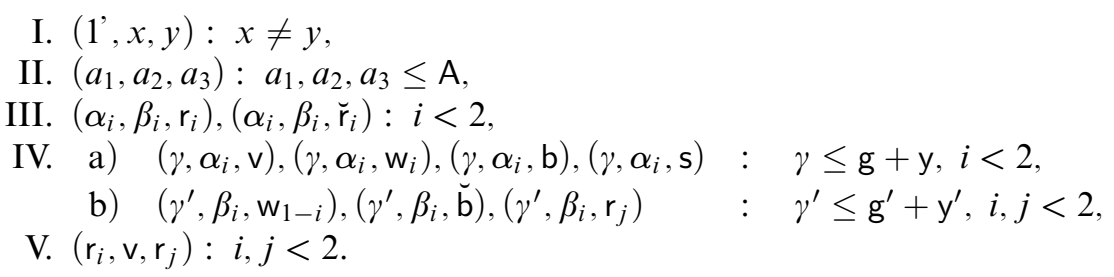

Rules II-V are illustrated in Figure 1. The set of forbidden triples is $F$. Let $C=A t^{3} \backslash F$ (the consistent triples). This defines the atom structure $A t$. We define $\mathscr{A}_{0}$ to be the complex algebra over $A t$. 


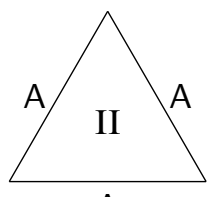

A
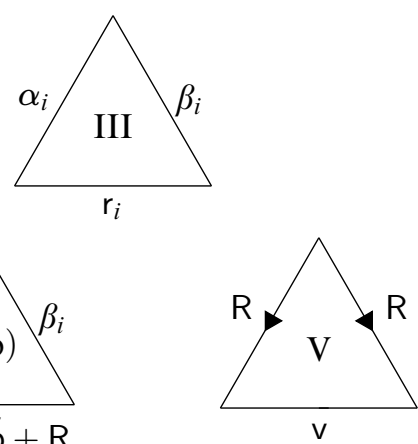

Figure 1. The main forbidden triples of $\mathscr{A}_{0}$.

LEMMA 13. $\mathscr{A}_{0}$ is a relation algebra.

Proof. By [4, Lemma 3.24], the following three properties prove that $\mathscr{A}_{0}$ is a relation algebra: (i) For all $x, y \in A t$ we have $x=y \Longleftrightarrow\left(1^{\prime}, x, y\right) \in C$, (ii) $C$ is closed under Peircean transforms and (iii) for all $b, c, d, e \in A t$,

$$
\exists a[(b, c, a),(a, e, d) \in C] \Longleftrightarrow \exists f[(b, f, d),(c, e, f) \in C] .
$$

The first two properties are true by definition of $C$. The third property is easily seen to be true if $1^{\prime} \in\{b, c, d, e\}$. For example, if $b=1^{\prime}$ then the only possibility for $a$ is $c$, the only possibility for $f$ is $d$, and both sides reduce to $(c, e, d) \in C$. If $1^{\prime} \notin\{b, c, d, e\}$ then both sides of the equivalence are true. This is because plainly, each rule can now eliminate at most four of the nine atoms beneath $\mathrm{E}$. Rule I is not applicable because $b, c, d, e \neq 1^{\prime}$ and $\mathrm{E} \cdot 1^{\prime}=0$. Rule IV(b) can eliminate four atoms beneath $\mathrm{E}$ (namely, $\mathrm{w}_{1-i}, \breve{\mathrm{b}}, \mathrm{r}_{0}, \mathrm{r}_{1}$ ), and each of the remaining rules can eliminate at most three. So for any $b, c, d, e$, the two conditions $(b, c, a),(a, e, d) \in C$ on the left of the equivalence can eliminate at most eight of the nine atoms $a \leq \mathrm{E}$, and so there is always a possible choice of $a \leq \mathrm{E}$ making the left-hand side true. Similarly, there is always a value of $f \leq \mathrm{E}$ making the right-hand side true too.

Lemma 14. $\mathscr{A}_{0}$ does not have a five-dimensional relational basis.

Proof. Suppose $B$ is a five-dimensional relational basis for $\mathscr{A}_{0}$, for contradiction. See Figure 2. Then there is $N_{0} \in B$ with $N_{0}(0,1)=v$. Since $(\mathrm{g}, \mathrm{y}, \mathrm{v})$ is not forbidden we have $\mathrm{g} ; \mathrm{y} \geq \vee$ so there must be $N_{1} \in B$ with $N_{1} \equiv_{2} N_{0}$ and $N_{1}(0,2)=\mathrm{g}$, $N_{1}(2,1)=\mathrm{y}$. Similarly, since $\left(\mathrm{g}^{\prime}, \mathrm{y}^{\prime}, \mathrm{v}\right)$ is not forbidden, there must be $N_{2} \in B$ with $N_{2} \equiv_{3} N_{1}, N_{2}(0,3)=\mathrm{g}^{\prime}$, and $N_{2}(3,1)=\mathrm{y}^{\prime}$. Note that $N_{2}(2,3) \cdot\left(1^{\prime}+\mathrm{A}\right)=0$, by forbidden triples I and II, and therefore

$$
N_{2}(2,3) \leq \mathrm{w}_{0}+\mathrm{w}_{1}+\mathrm{b}+\breve{\mathrm{b}}+\mathrm{s}+\mathrm{v}+\mathrm{r}_{i}+\breve{\mathrm{r}}_{i}
$$

for some $i<2$.

Write $j$ for $1-i$. It can be checked that $\left(\alpha_{j}, \beta_{j}, N_{2}(2,3)\right)$ is not forbidden by any of the rules defining forbidden triples, so there is $N_{3} \in B$ with $N_{3} \equiv_{4} N_{2}$ and $N_{3}(2,4)=\alpha_{j}, N_{3}(4,3)=\beta_{j}$. Then $N_{3}(0,4) \leq \mathrm{g} ; \alpha_{j} \cdot \mathrm{g}^{\prime} ; \beta_{j}=\breve{\mathrm{R}}$ (by I, II and IV) and similarly, $N_{3}(1,4) \leq \mathrm{y} ; \alpha_{j} \cdot \mathrm{y}^{\prime} ; \beta_{j}=\breve{\mathrm{R}}$. But then $N_{3}(0,4)=$ 
$N_{3}(0,1) ; N_{3}(1,4) \cdot N_{3}(0,4) \leq \mathrm{v} ; \breve{\mathrm{R}} \cdot \breve{\mathrm{R}}=0$, by $\mathrm{V}$. This is a contradiction and proves that a relational basis does not exist.

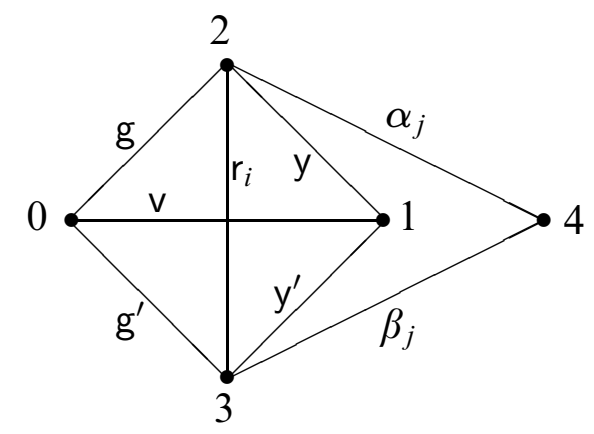

FIGURE 2. Impossible to complete a labelling by giving a consistent label to $(4,0),(4,1)$. The edge $(2,3)$ has no arrow, as both $r_{i}$ and $\breve{\mathrm{r}}_{i}$ (as well as $\mathrm{w}_{0}, \mathrm{w}_{1}, \mathrm{~b}, \breve{\mathrm{b}}, \mathrm{s}, \mathrm{v}$ ) are possible.

THEOREM $15 . \mathscr{A}_{0}$ is weakly representable.

Proof. We show that $\exists$ has a winning strategy in the weak representation game $\mathscr{G}\left(\mathscr{A}_{0}\right)$ (see Definition 9). Suppose that $\forall$ selects $a_{0} \in \mathscr{A}_{0} \backslash\{0\}$ in his initial move. $\exists$ responds with a strict atomic network $N_{0}=\left(\left\{x_{0}, y_{0}\right\}, l\right)$, where $l\left(x_{0}, y_{0}\right)$ is an arbitrary atom beneath $a_{0}$, and $x_{0}=y_{0}$ iff $l\left(x_{0}, y_{0}\right)=1$ '. This determines $N_{0}$.

Suppose at some point in a play of the game that the current network is $N$. An edge of $N$ is an ordered pair of nodes of $N$. An edge $(r, s)$ of $N$ is said to be critical (in $N$ ) if $r \neq s$ and there are nodes $t, u$ of $N$ such that $N(r, t), N(r, u), N(s, t), N(s, u) \leq \Gamma$ and $N(t, u)=\mathrm{v}$. We will assume inductively that for every $r, s \in N$ :

I1) If $(r, s)$ is non-critical then $N(r, s) \in \operatorname{At}\left(\mathscr{A}_{0}\right)$.

I2) If $(r, s)$ is critical then $N(r, s) \in\{\mathrm{R}, \breve{\mathrm{R}}\}$. See Figure 3.

I3) Every atomic refinement of $N$ is a strict network.

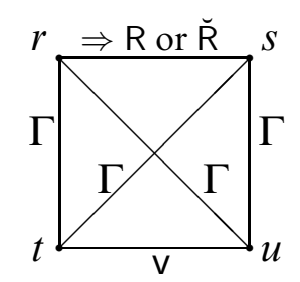

Figure 3. Critical edge $(r, s)$ of $N$.

Clearly, these assumptions hold for the initial network $N_{0}$. Assume that they hold for $N$. Let $\forall$ 's move in the current round of the game be $(m, n, Y, Z)$, where $m, n \in N$ and $Y ; Z \geq N(m, n)$. Nodes $m, n \in N$ and elements $Y, Z \in \mathscr{A}_{0}$ are fixed for the duration of the proof of this theorem. $\exists$ 's job is to find a network 
$N^{+} \geq N$ satisfying the three inductive conditions and containing a node $p$ with $N^{+}(m, p) \leq Y$ and $N^{+}(p, n) \leq Z$. This is not so hard. We will now explain how she can do it.

First of all, if $N$ already contains a node $p$ with $N(m, p) \leq Y$ and $N(p, n) \leq Z$, then $\exists$ may and does respond to $\forall$ 's move by playing $N^{+}=N$. This obviously keeps all the inductive conditions, and it completes the round of the game in this case. So assume from now on that there is no such $p$ in $N$.

Claim. There are atoms $b \leq Y, c \leq Z$ such that $b ; c \geq N(m, n)$.

Proof OF CLAIM. If $(m, n)$ is non-critical then $N(m, n)$ is already an atom of $\mathscr{A}_{0}$, so such atoms $b, c$ exist by additivity of ';' in $\mathscr{A}_{0}$. If $(m, n)$ is critical, then $N(m, n)$ is $\mathrm{R}$ or $\breve{\mathrm{R}}$. Suppose that $N(m, n)=\mathrm{R}$ (the case where $N(m, n)=\breve{\mathrm{R}}$ is similar). Suppose for contradiction that no suitable $b, c$ exist. Since $Y ; Z \geq \mathrm{R}=\mathrm{r}_{0}+\mathrm{r}_{1} \geq \mathrm{r}_{0}$, there are atoms $b_{0} \leq Y, c_{0} \leq Z$ with $b_{0} ; c_{0} \geq \mathrm{r}_{0}$, and therefore by hypothesis, $b_{0} ; c_{0} \geq \mathrm{r}_{1}$. Similarly, there are atoms $b_{1} \leq Y, c_{1} \leq Z$ with $b_{1} ; c_{1} \geq \mathrm{r}_{1}$ but $b_{1} ; c_{1} \geq \mathrm{r}_{0}$. Referring back to the list of forbidden triples, we see that only rules I and III distinguish between $r_{0}$ and $r_{1}$, so it follows that $\left(b_{0}, c_{0}\right) \in\left\{\left(1^{\prime}, r_{0}\right),\left(r_{0}, 1^{\prime}\right),\left(\alpha_{1}, \beta_{1}\right),\left(\beta_{1}, \alpha_{1}\right)\right\}$ and $\left(b_{1}, c_{1}\right) \in\left\{\left(1^{\prime}, r_{1}\right),\left(r_{1}, 1^{\prime}\right),\left(\alpha_{0}, \beta_{0}\right),\left(\beta_{0}, \alpha_{0}\right)\right\}$. The 16 possibilities are tabulated in Figure 4. Entries in the body of the table are of two kinds. Fourteen of them

\begin{tabular}{|c|cccc|}
\hline$b_{0}, c_{0} \backslash b_{1}, c_{1}$ & $1^{\prime}, \mathrm{r}_{1}$ & $\mathrm{r}_{1}, 1^{\prime}$ & $\alpha_{0}, \beta_{0}$ & $\beta_{0}, \alpha_{0}$ \\
\hline $1^{\prime}, \mathrm{r}_{0}$ & Id-move & $\mathrm{r}_{1}, \mathrm{r}_{0}$ & $\alpha_{0}, \mathrm{r}_{0}$ & $\beta_{0}, \mathrm{r}_{0}$ \\
$\mathrm{r}_{0}, 1^{\prime}$ & $\mathrm{r}_{0}, \mathrm{r}_{1}$ & Id-move & $\mathrm{r}_{0}, \beta_{0}$ & $\mathrm{r}_{0}, \alpha_{0}$ \\
$\alpha_{1}, \beta_{1}$ & $\alpha_{1}, \mathrm{r}_{1}$ & $\mathrm{r}_{1}, \beta_{1}$ & $\alpha_{0}, \beta_{1}$ & $\alpha_{1}, \alpha_{0}$ \\
$\beta_{1}, \alpha_{1}$ & $\beta_{1}, \mathrm{r}_{1}$ & $\mathrm{r}_{1}, \alpha_{1}$ & $\alpha_{0}, \alpha_{1}$ & $\beta_{1}, \alpha_{0}$ \\
\hline
\end{tabular}

Figure 4. Atoms $b_{0}, c_{0}, b_{1}, c_{1}$.

consist of a pair of atoms, $b, c$, say. It can be checked that in each case, $b \in$ $\left\{b_{0}, b_{1}\right\}$ and so $b \leq Y, c \in\left\{c_{0}, c_{1}\right\}$ and so $c \leq Z$, and $b ; c \geq \mathrm{R}$, contrary to our hypothesis that no such atoms exist. So we are in fact in one of the two remaining cases 'Id-move': either $b_{0}=b_{1}=1$ ' and $c_{i}=\mathrm{r}_{i}$ for each $i$, or the other way round, swapping $b, c$. But then, $N$ already contains a node $p$ with $N(m, p) \leq Y$ and $N(p, n) \leq Z$, namely $p=m$ (or $p=n$ when $b, c$ are swapped). This contradicts our assumption above that there is no such $p$ in $N$, and proves the claim.

$\exists$ chooses atoms $b, c$ as in the claim. If $b=1^{\prime}$ or $c=1^{\prime}$, then again she has the trivial response of $N^{+}=N$ to $\forall$ 's move, contrary to assumption. So this is not the case. $\exists$ now begins the construction of $N^{+}$, by creating a new node $p \notin N$. The nodes of $N^{+}$will be those of $N$ together with $p$. She defines $N^{+}(m, p)=b$ and $N^{+}(p, n)=c$. Further, she lets $N^{+}(p, m)=\breve{b}$ and $N^{+}(n, p)=\breve{c}$. This is well defined in the case where $m=n$ : for then, $b ; c \geq N(m, m)=1$ ', so by rule I, $b=\breve{c}$. She sets $N^{+}(p, p)=1^{\prime}$ of course.

It remains for her to define $N^{+}(p, q)$ and $N^{+}(q, p)$ for each $q \in N \backslash\{m, n\}$. She will define them to be mutually converse, so it is enough to specify either one. Her strategy is as follows: 

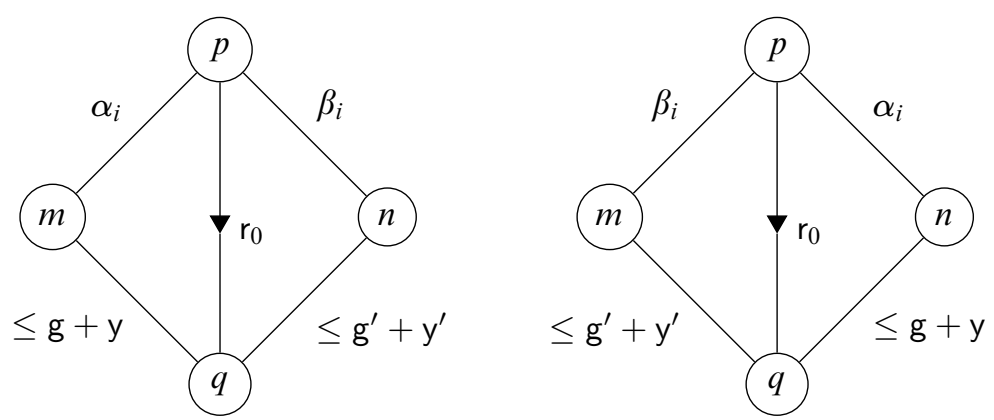

Figure 5. Case S3 of $\exists$ 's strategy.

S1) If $N(q, m), N(q, n), b, c \leq \Gamma$ and $N(m, n)=\mathrm{v}$, then define $N^{+}(q, p)=\mathrm{R}$.

S2) Otherwise, if there is $a \leq \mathrm{w}_{0}+\mathrm{w}_{1}+\mathrm{b}+\breve{\mathrm{b}}+\mathrm{s}+\mathrm{R}$ such that for every atomic refinement $N^{a}$ of $N$, the triples $\left(N^{a}(q, m), b, a\right)$ and $\left(N^{a}(q, n), \breve{c}, a\right)$ are not forbidden, then choose any such $a$ and set $N^{+}(q, p)=a$. (For definiteness, we could let $a$ be the first applicable of the atoms $w_{0}, w_{1}, b, \breve{b}, s, r_{0}, r_{1}$ in that order.)

S3) Otherwise, define $N^{+}(p, q)=\mathrm{r}_{0}$. ( $\mathrm{r}_{1}$ would also do.)

This is plainly well defined and completes the definition of $N^{+}$. Note that S1-S3 only select elements beneath E. (Broadly, elements below E are most useful for $\exists$, and those below $A$ are most useful for $\forall$.)

Before we proceed, we make two remarks.

REMARK 16. Let us analyse case S3 of the strategy a little. Suppose that $\exists$ used $\mathrm{S} 3$, so that $N^{+}(p, q)=\mathrm{r}_{0}$. So S2 does not apply, and therefore all seven atoms $\mathrm{w}_{0}$, $\mathrm{w}_{1}, \mathrm{~b}, \breve{\mathrm{b}}, \mathrm{s}, \mathrm{r}_{0}$, and $\mathrm{r}_{1}$ are forbidden by some rules and atomic refinements of $N$ in the triangles $(q, m, p),(q, n, p)$.

How can this be? Atomic refinements differ from $N$ only by having labels $\mathrm{r}_{0}$ or $r_{1}$ instead of $\mathrm{R}$ (or with converses). Examination of the list of forbidden triples shows that even allowing for various different atomic refinements, the only rules that can forbid more than three of the seven atoms are rules I and IV(b). Rule I is not involved, since (inductively) every atomic refinement of $N$ is strict, we assumed $b, c \neq 1^{\prime}$, and none of $\mathrm{w}_{0}, \mathrm{w}_{1}, \mathrm{~b}, \breve{\mathrm{b}}, \mathrm{s}, \mathrm{r}_{0}, \mathrm{r}_{1}$ are $1^{\prime}$. Therefore, $\mathrm{IV}(\mathrm{b})$ must be involved, and either $b$ or $c$ is $\beta_{i}$ for some $i<2$. Suppose first that $c=\beta_{i}$, and $N(q, n) \leq \mathrm{g}^{\prime}+\mathrm{y}^{\prime}$. Now, $\mathrm{w}_{1-i}, \breve{\mathrm{b}}, r_{0}, \mathrm{r}_{1}$ are forbidden on $(q, p)$ by dint of the triangle $(q, n, p)$. So the remaining atoms $\mathrm{w}_{i}, \mathrm{~b}, \mathrm{~s}$ are being forbidden by the other triangle, $(q, m, p)$. The only rule that can do the job is $\operatorname{IV}(\mathrm{a})$, and its 'orientation' must be the same as for $\mathrm{IV}(\mathrm{b})$ : we must have $b=\alpha_{i}$ (the same $i$ as for $\beta_{i}$ ) and $N(q, m) \leq \mathrm{g}+\mathrm{y}$. We have arrived at the situation shown on the left of Figure 5. The other possibility, shown on the right, is obtained when $b=\beta_{i}$.

REMARK 17. Observe that no edge of $N$ changes its criticality when viewed as an edge of $N^{+}$. Certainly, being critical is an existential condition and cannot be lost from $N$ to $N^{+}$. Suppose for contradiction that $(r, s)$ is non-critical in $N$ but critical in $N^{+}$. Then there are nodes $t, u$ of $N^{+}$as in Figure 3. Such nodes did not exist in $N$, so $p \in\{t, u\}$. But this means that there are three edges of 
$N^{+}$involving $p$ and labelled with atoms $\leq \Gamma+\mathrm{v}$. At most two of them can be edges from $p$ to $m$ or $n$, so at least one of them was labeled by $\exists$ according to $\mathrm{S} 1-\mathrm{S} 3$ of her strategy. Since $\mathrm{S} 1-\mathrm{S} 3$ never use atoms $\leq \Gamma+\mathrm{v}$, this is impossible.

Now let us proceed to check that $N^{+}$satisfies the inductive conditions. We need to check that if $(r, s)$ is non-critical then $N^{+}(r, s)$ is an atom, and if it is critical then $N^{+}(r, s)$ is R or $\breve{\mathrm{R}}$. By the inductive hypothesis and Remark 17, we only need consider edges involving the new node, $p$. Since always $N^{+}(q, p)=N^{+}(p, q)^{\smile}$, we only need consider one orientation.

- If $p=q$ then $(p, q)$ is non-critical and $N^{+}(p, q)=1$ ', an atom.

- The edge $(m, p)$ is labeled by an atom ( $b$, by construction). Also, it is not critical in $N^{+}$. For otherwise, there would be distinct nodes $t, u \in N \backslash\{m\}$ with $N^{+}(p, t), N^{+}(p, u) \leq \Gamma$ (see Figure 3). As in Remark 17, at least one of them was labeled by $\exists$ using S1-S3 of her strategy, which is impossible since $\mathrm{S} 1-\mathrm{S} 3$ do not use atoms $\leq \Gamma$.

- The edge $(p, n)$ is handled similarly.

- Now let $q \in N \backslash\{m, n\}$. The only occasion when $\exists$ uses a non-atom $(\breve{\mathrm{R}})$ to label $(p, q)$ is in case $\mathrm{S} 1$, and that is exactly when $(p, q)$ is critical in $N^{+}$. Certainly, if $\exists$ uses $\breve{R}$ then by $\mathrm{S} 1,(p, q)$ is critical. Conversely, if $(p, q)$ is critical then there are distinct $t, u \in N$ as in Figure 3, with $N^{+}(p, t), N^{+}(p, u) \leq \Gamma$. Since S1-S3 never use atoms $\leq \Gamma$, we must have $\{t, u\}=\{m, n\}$. Therefore, $b, c, N(m, q), N(n, q) \leq \Gamma$ and $N(m, n)=\mathrm{v}$. So S1 applies and $\exists$ defines $N^{+}(p, q)=\breve{\mathrm{R}}$.

So inductive requirements $\mathrm{I} 1$ and $\mathrm{I} 2$ hold for $N^{+}$. The main work is to check I3: that every atomic refinement of $N^{+}$is a strict network. Let $N^{a}$ be such a refinement. It is clearly strict, as (inductively) every atomic refinement of $N$ is strict, and because $b, c \neq 1$ ' (by assumption), the only edge that $\exists$ labels with an $\mathscr{A}_{0}$-element $\geq 1^{\prime}$ is $(p, p)$. So we need to check that for no $x, y, z \in N^{+}$ is $\left(N^{a}(x, y), N^{a}(y, z), N^{a}(x, z)\right)$ a forbidden triple. The restriction of $N^{a}$ to the nodes of $N$ is an atomic refinement of $N$, so the condition holds inductively if $x, y, z \in N$. It is easily seen to hold if $x, y, z$ are not all distinct. So we can assume that $p \in\{x, y, z\}$ and $|\{x, y, z\}|=3$. Because the labels on edges of $N^{a}$ are atoms and the forbidden triples are closed under Peircean transforms, the order of $x, y, z$ is not significant. (This is why atomic refinements are helpful.)

We now divide into cases according to whether the set $\{x, y, z\} \cap\{m, p, n\}$ has size 3, 2, or 1 . If $(x, y, z)=(m, p, n)$, then $N^{a}(x, y)=b$ and $N^{a}(y, z)=c$. We know that $b ; c \geq N(m, n) \geq N^{a}(x, z)$, and we are done.

Suppose that $x \in N \backslash\{m, n\}, y \in\{m, n\}$, and $z=p$. If $\exists$ used case S1 of her strategy to define $N^{+}(x, p)=\mathrm{R}$, then we have $N^{a}(x, y), N^{a}(y, p) \leq \Gamma$, and $N^{a}(x, p) \in\left\{r_{0}, r_{1}\right\}$. No forbidden triple involves $\Gamma, \Gamma, \mathrm{R}$, so we are done. If she used $\mathrm{S} 2$, she defined $N^{+}(q, p)$ to be some atom $a \leq \mathrm{w}_{0}+\mathrm{w}_{1}+\mathrm{b}+\breve{\mathrm{b}}+\mathrm{s}+\mathrm{R}$ precisely so that the triple $\left(N^{a}(x, y), N^{a}(y, p), N^{a}(x, p)\right)$ is not forbidden. If she used S3, so that $N^{a}(x, p)=\breve{\mathrm{r}}_{0}$, then we are in the position of Figure 5 with $x=q$, from which it is clear that neither $\left(N^{a}(q, m), N^{a}(m, p), \breve{r}_{0}\right)$ nor $\left(N^{a}(q, n), N^{a}(n, p), \breve{r}_{0}\right)$ are forbidden. 
The last case is when $x, y \in N \backslash\{m, n\}$ are distinct and $z=p$. Therefore, by S1-S3,

$$
N^{a}(x, z), N^{a}(y, z) \leq \mathrm{E} .
$$

Assume for contradiction that $\left(N^{a}(x, y), N^{a}(y, z), N^{a}(x, z)\right)$ is a forbidden triple. The only rules that can forbid a triple with two of its entries under $\mathrm{E}$ are rules I and V. Rule I is not applicable because none of $N^{a}(x, y), N^{a}(y, z), N^{a}(x, z)$ are 1'. For rule $\mathrm{V}$ to be applicable, by (5) we must have $N^{+}(p, x), N^{+}(p, y) \leq$ $\mathrm{R}$, and $N^{+}(x, y)=\mathrm{v}$. That is, $\exists$ used case S3 of her strategy to label both $(p, x),(p, y)$. Referring to Remark 16 and Figure 5 again, we have $(b, c) \in$ $\left\{\left(\alpha_{i}, \beta_{i}\right),\left(\beta_{i}, \alpha_{i}\right)\right\}$ for some $i<2$; by symmetry, we can suppose $b=\alpha_{i}, c=\beta_{i}$. Then (see Figure 5) we have $N(m, x), N(m, y) \leq \mathrm{g}+\mathrm{y} \leq \Gamma$ and $N(n, x), N(n, y) \leq$ $\mathrm{g}^{\prime}+\mathrm{y}^{\prime} \leq \Gamma$. This is summarised in Figure 6 , from which we see that $(m, n)$ must be a critical edge of $N$. Therefore, by inductive condition $\mathrm{I} 2, N(m, n) \in$ $\{\mathrm{R}, \breve{\mathrm{R}}\}$. But by rule III, this means that $N(m, n) \not \leq \alpha_{i} ; \beta_{i}=b ; c$, which is a contradiction.

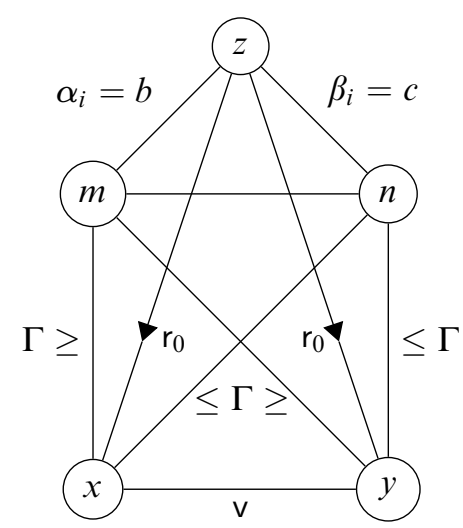

Figure 6. Last case of I3.

We have verified that inductive conditions I1-I3 hold for $N^{+}$. It follows that $N^{+}$ is a network. For let $x, y, z \in N^{+}$. Take any atom $a \leq N^{+}(x, z)$, and any atomic refinement $N^{a}$ of $N^{+}$with $N^{a}(x, z)=a$. We have proved that $N^{a}$ is a network, so $a \leq N^{a}(x, y) ; N^{a}(y, z) \leq N^{+}(x, y) ; N^{+}(y, z)$. This holds for all such $a$, and so $N^{+}(x, z) \leq N^{+}(x, y) ; N^{+}(y, z)$ as required.

Thus $N^{+}$is a network extending $N$ with $N^{+}(m, p)=b \leq Y$ and $N^{+}(p, n)=$ $c \leq Z$. Since $\exists$ can always respond to $\forall$ 's move with a suitable network, she is sure to win the game. Hence, by Proposition $10, \mathscr{A}_{0}$ has a weak representation.

Problem 18. Let $n \geq 5$ and $K_{n}=w_{R R A} \cap R$. Prove the following conjecture: $K_{n}$ cannot be defined over wRRA using only finitely many axioms, nor can it be defined over $\mathrm{RA}_{n}$ using only finitely many axioms. 


\section{REFERENCES}

[1] H. ANDRÉKA, Weakly representable but not representable relation algebras, Algebra Universalis, vol. 32 (1994), pp. 31-43.

[2] M. Haiman, Arguesian lattices which are not linear, Bulletin of the American Mathematical Society, vol. 16 (1987), pp. 121-123.

[3] - Arguesian lattices which are not type I, Algebra Universalis, vol. 28 (1991), pp. 128-137.

[4] R. HiRSCH and I. HodKINSON, Relation algebras by games, Studies in Logic and the Foundations of Mathematics, vol. 147, North-Holland, Amsterdam, 2002.

[5] I. Hodkinson and Sz. Mikulás, Non-finite axiomatizability of reducts of algebras of relations, Algebra Universalis, vol. 43 (2000), pp. 127-156.

[6] I. Hodkinson and Y. Venema, Canonical varieties with no canonical axiomatisation, Transactions of the American Mathematical Society, vol. 357 (2005), pp. 4579-4605.

[7] B. JóNSSON, Representation of modular lattices and of relation algebras, Transactions of the American Mathematical Society, vol. 92 (1959), pp. 449-464.

[8] — , The theory of binary relations, Algebraic logic (H. Andréka, J. D. Monk, and I. Németi, editors), Colloq. Math. Soc. J. Bolyai, vol. 54, North-Holland, Amsterdam, 1991, pp. 245-292.

[9] R. LyNDON, The representation of relational algebras, Annals of Mathematics, vol. 51 (1950), no. 3, pp. 707-729.

[10] R. D. Maddux, Some varieties containing relation algebras, Transactions of the American Mathematical Society, vol. 272 (1982), no. 2, pp. 501-526.

[11] — A sequent calculus for relation algebras, Annals of Pure and Applied Logic, vol. 25 (1983), pp. 73-101.

[12] - Relation algebras of every dimension, this JourNAL, vol. 57 (1992), pp. 1213-1229.

[13] — Relation algebras, Studies in Logic and the Foundations of Mathematics, vol. 150, Elsevier, Amsterdam, 2006.

[14] J. D. Monk, On representable relation algebras, Michigan Mathematics Journal, vol. 11 (1964), pp. 207-210.

[15] B. PÉCSI, Weakly representable relation algebras form a variety, Algebra Universalis, vol. 60 (2009), pp. 369-380.

[16] A. TARSKI and S. R. GIVANT, A formalization of set theory without variables, Colloquium Publications, no. 41, American Mathematical Society, Providence, Rhode Island, 1987.

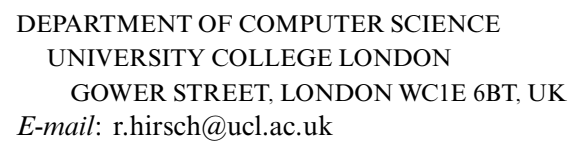

E-mail: i.hodkinson@imperial.ac.uk

DEPARTMENT OF MATHEMATICS

IOWA STATE UNIVERSITY

AMES, IA 50011, USA

E-mail:maddux@iastate.edu 